\title{
An analytical decomposition protocol for optimal implementation of two-qubit entangling gates
}

\author{
M Blaauboer and $\mathbf{R} \mathbf{L}$ de Visser \\ Kavli Institute of Nanoscience, Delft University of Technology, Lorentzweg 1, 2628 CJ Delft, \\ The Netherlands
}

Received 2 April 2008, in final form 28 July 2008

Published 5 September 2008

Online at stacks.iop.org/JPhysA/41/395307

\begin{abstract}
This paper addresses the question of how to implement a desired two-qubit gate $U$ using a given tunable two-qubit entangling interaction $\mathcal{H}_{\text {int }}(t)$. We present a general method which is based on the $K_{1} A K_{2}$ decomposition of unitary matrices $\in S U(4)$ to calculate the smallest number of two-qubit gates $U_{\text {int }}(t)$ (based on $\mathcal{H}_{\text {int }}(t)$ ) and single-qubit rotations, and the explicit sequence of these operations that are required to implement $U$. We illustrate our protocol by calculating the implementation of (1) the transformation from standard basis to Bell basis, (2) the CNOT-gate and (3) the quantum Fourier transform for two kinds of interaction-Heisenberg exchange interaction and quantum inductive coupling - and discuss the relevance of our results for solid-state qubits.
\end{abstract}

PACS numbers: $03.67 . \mathrm{Lx}$, 03.67.Mn, 73.21.La, 85.25.Cp

\section{Introduction}

How to implement two-qubit quantum circuits in an optimal way, by which we mean here that they require a minimum number of operations, has become a topic of active interest over the last few years. Typically, the central question asked is how to perform an arbitrary operation $\in S U$ (4) with the least number of two-qubit gates such as the CNOT (controlled NOT) gate. This has led to the discovery that any two-qubit operation can be implemented using three CNOT-gates and local gates in between [1]. Alternatively, two-qubit operations can be constructed by three applications of the (SWAP) ${ }^{\alpha}$ gate [2], with $0<\alpha \leqslant 1$, or by two applications of the B-gate [3]. From a practical point of view, it is essential to perform quantum operations as fast as possible, before decoherence (due to interactions with the environment) sets in. This is of particular importance for solid-state qubits, which in general interact strongly with their environment. For a specific physical system, however, efficient implementation of quantum operations does not necessarily coincide with minimizing the number of CNOT-gates, 
if CNOT cannot be directly generated by the given two-qubit Hamiltonian. Rather, efficient construction of quantum operations requires minimizing the number of two-qubit gates which are based on the (entangling) two-qubit interaction that is naturally available in the system, as well as minimizing the number of single qubit operations (which in practice generally also take a finite amount of time to implement ${ }^{1}$ ).

In this paper, we present a general, explicit and self-contained analytical protocol for calculating the implementation of a desired two-qubit quantum gate $U \in S U(4)$ as a sequence of single-qubit rotations and two-qubit gates $U_{\text {int }}(t)$, where $U_{\text {int }}(t)$ is based on the two-qubit interaction $\mathcal{H}_{\text {int }}(t)$ that is naturally available in a given system, $U_{\text {int }}(t) \equiv$ $\exp \left[-\frac{\mathrm{i}}{\hbar} \int_{0}^{t} \mathrm{~d} t^{\prime} \mathcal{H}_{\text {int }}\left(t^{\prime}\right)\right]$. We assume that $U_{\text {int }}(t)$ has some entangling capacity, which is a necessary requirement if $U$ itself is an entangling gate. We also assume that $U_{\text {int }}(t)$ is a tunable gate, i.e. that one can switch the interaction $\mathcal{H}_{\text {int }}(t)$ in the system on and off during well-defined amounts of time by changing external parameters. This is often the case in real qubit systems where the qubits consist of massive particles such as electrons or ions. Our scheme is based on separating the two-qubit (entangling) part of $U$ from the single-qubit basis transformations (the so-called $K_{1} A K_{2}$ or single-value decomposition [4, 5], with $A \in S U$ (4) and $\left.K_{1}, K_{2} \in S U(2) \times S U(2)\right)$, translating each of the matrices $K_{1}, A$, and $K_{2}$ into a sequence of operations which involves the smallest possible number of $U_{\text {int }}(t)$ for a suitably chosen time $t$, and minimizing the number of single-qubit rotations in the total sequence of operations using permutation relations. The resulting shortest-sequence implementation of $U$-which we refer in this paper to as the optimal implementation of $U$-contains the least number of operations from the given 'library' of single- and two-qubit gates ${ }^{2}$. We illustrate our scheme by calculating the optimal implementation of three elementary quantum operations $U$ (the transformation from standard basis to Bell basis, the CNOT-gate, and the quantum Fourier transform) for two kinds of two-qubit interaction $\mathcal{H}_{\text {int }}$ that are relevant for solid-state qubits: Heisenberg exchange interaction (corresponding to the (SWAP) ${ }^{\alpha}$-gate) and quantum inductive coupling (corresponding to the B-gate). Either of these gates, in combination with single-qubit rotations, forms a so-called universal set $[2,3,7]$ into which any operator $U \in S U(4)$ can be decomposed.

This paper is organized as follows: in section 2 we outline the decomposition protocol, starting with the decomposition of an arbitrary gate $U \in S U(4)$ into matrices $K_{1}, A$ and $K_{2}$. Section 2.2 addresses the special case in which $U$ is a maximally entangling gate, for which the protocol can be simplified. In section 2.3 we show how each of the matrices $K_{1}, A$ and $K_{2}$ can be decomposed into a sequence of operations consisting of single-qubit rotations and a minimum number of two-qubit interactions $U_{\text {int }}(t)$ of a given kind. In section 2.4 we show how to iteratively minimize the total number of single-qubit rotations in the decomposition of $U$, and thereby obtain the implementation of $U$ that involves the smallest number of both $U_{\text {int }}(t)$ and single-qubit rotations. In section 3, we illustrate the decomposition protocol for two kinds of interaction that occur in solid-state qubit systems: Heisenberg exchange interaction $\mathcal{H}_{E X}$ and quantum inductive coupling $\mathcal{H}_{\text {ind }}$. We show how to implement the transformation from standard basis to Bell basis (section 3.1), the CNOT-gate (section 3.2) and the quantum Fourier transform (section 3.3) using either of these interactions. Finally, in section 4 we discuss how our results can be implemented for electron spin qubits in quantum dots and for superconducting flux qubits, followed by conclusions in section 5.

\footnotetext{
1 For some qubit systems, in particular solid-state systems, the time required for implementing single-qubit gates is currently longer than for two-qubit operations, see e.g. [24, 25].

2 A systematic method of calculating the time-optimal implementation of a given $U$ in terms of a given $U_{\text {int }}(t)$, assuming instantaneous single-qubit operations (local unitaries), has been given by Vidal et al [6].
} 


\section{An explicit decomposition protocol}

\subsection{General gates $U \in S U(4)$}

The decomposition protocol we present in this section is based on the $K_{1} A K_{2}$ decomposition [4, 5], which states that every two-qubit operation $U \in S U(4)$ can be written as $U=K_{1} A K_{2}$, with $K_{1}, K_{2} \in S U(2) \otimes S U(2)$ and

$$
\begin{aligned}
A & =\exp \left[\mathrm{i}\left(\alpha \sigma_{x} \otimes \sigma_{x}+\beta \sigma_{y} \otimes \sigma_{y}+\gamma \sigma_{z} \otimes \sigma_{z}\right)\right] \\
& =\left(\begin{array}{cccc}
P_{\alpha \beta}^{-} \mathrm{e}^{\mathrm{i} \gamma} & 0 & 0 & \mathrm{i} Q_{\alpha \beta}^{-} \mathrm{e}^{\mathrm{i} \gamma} \\
0 & P_{\alpha \beta}^{+} \mathrm{e}^{-\mathrm{i} \gamma} & \mathrm{i} Q_{\alpha \beta}^{+} \mathrm{e}^{-\mathrm{i} \gamma} & 0 \\
0 & \mathrm{i} Q_{\alpha \beta}^{+} \mathrm{e}^{-\mathrm{i} \gamma} & P_{\alpha \beta}^{+} \mathrm{e}^{-\mathrm{i} \gamma} & 0 \\
\mathrm{i} Q_{\alpha \beta}^{-} \mathrm{e}^{\mathrm{i} \gamma} & 0 & 0 & P_{\alpha \beta}^{-} \mathrm{e}^{\mathrm{i} \gamma}
\end{array}\right)
\end{aligned}
$$

with $P_{\alpha \beta}^{ \pm} \equiv \cos (\alpha \pm \beta)$ and $Q_{\alpha \beta}^{ \pm} \equiv \sin (\alpha \pm \beta)$. Here $\alpha, \beta, \gamma \in[0,2 \pi)$ and $\sigma_{x}, \sigma_{y}, \sigma_{z}$ denote the Pauli matrices. Our goals in this section are: (1) to calculate the matrices $K_{1}, K_{2}$ and $A$ for a given $U \in S U(4)$ and (2) to translate each of these matrices into the smallest number of single-qubit rotations and two-qubit interactions $U_{\text {int }}(t)$ of a given kind. In this subsection and the next we develop a systematic protocol for the first goal ${ }^{3}$. The implementation of $K_{1}, A$ and $K_{2}$ using $U_{\text {int }}(t)$ and single-qubit rotations is the subject of section 2.3. We begin by introducing the matrix $Q \in S U(4)$ defined as

$$
Q \equiv \frac{1}{\sqrt{2}}\left(\begin{array}{cccc}
1 & 0 & 0 & \mathrm{i} \\
0 & \mathrm{i} & 1 & 0 \\
0 & \mathrm{i} & -1 & 0 \\
1 & 0 & 0 & -\mathrm{i}
\end{array}\right)
$$

$Q$ transforms the standard basis $\left\{\left|e_{1}\right\rangle,\left|e_{2}\right\rangle,\left|e_{3}\right\rangle,\left|e_{4}\right\rangle\right\}$ of $S U(4)$ into the magic basis $\left\{\left|m_{1}\right\rangle,\left|m_{2}\right\rangle,\left|m_{3}\right\rangle,\left|m_{4}\right\rangle\right\}$. Here the standard basis is defined in spin-1/2 notation with the spin along the $z$-direction as $\left|e_{1}\right\rangle \equiv|\uparrow \uparrow\rangle,\left|e_{2}\right\rangle \equiv|\uparrow \downarrow\rangle,\left|e_{3}\right\rangle \equiv|\downarrow \uparrow\rangle,\left|e_{4}\right\rangle \equiv|\downarrow \downarrow\rangle$ and the magic basis is defined as:

$$
\begin{aligned}
\left|m_{1}\right\rangle & \equiv \frac{1}{\sqrt{2}}(|\uparrow \uparrow\rangle+|\downarrow \downarrow\rangle), \\
\left|m_{2}\right\rangle & \equiv \frac{1}{\sqrt{2}} \mathrm{i}(|\uparrow \downarrow\rangle+|\downarrow \uparrow\rangle), \\
\left|m_{3}\right\rangle & \equiv \frac{1}{\sqrt{2}}(|\uparrow \downarrow\rangle-|\downarrow \uparrow\rangle) \\
\left|m_{4}\right\rangle & \equiv \frac{1}{\sqrt{2}} \mathrm{i}(|\uparrow \uparrow\rangle-|\downarrow \downarrow\rangle) .
\end{aligned}
$$

Next, we define

$$
\begin{aligned}
& O_{1} \equiv Q^{\dagger} K_{1} Q \\
& O_{2} \equiv Q^{\dagger} K_{2} Q \\
& F \equiv Q^{\dagger} A Q
\end{aligned}
$$

where $O_{1}, O_{2} \in S O(4)[8]$ and $F=\operatorname{diag}\left(\lambda_{1}, \lambda_{2}, \lambda_{3}, \lambda_{4}\right)$ with $\lambda_{1}, \ldots, \lambda_{4}, \in \mathbb{C}$. Using (1), (2) and (6), we find that the relation between $\lambda_{1}, \ldots, \lambda_{4}$ and $\alpha, \beta, \gamma$ is

3 A similar decomposition procedure has been given in appendix A of [4]. In contrast to the latter, however, we derive explicit expressions for the matrices $K_{1}, A$ and $K_{2}$. 


$$
\begin{aligned}
& \lambda_{1}=\mathrm{e}^{\mathrm{i}(\alpha-\beta+\gamma)} \\
& \lambda_{2}=\mathrm{e}^{\mathrm{i}(\alpha+\beta-\gamma)} \\
& \lambda_{3}=\mathrm{e}^{\mathrm{i}(-\alpha-\beta-\gamma)} \\
& \lambda_{4}=\mathrm{e}^{\mathrm{i}(-\alpha+\beta+\gamma)} .
\end{aligned}
$$

Our aim ${ }^{4}$ is to find the matrices $O_{1}, O_{2}$ and $F$, and from those via (4)-(6) the matrices $K_{1}, K_{2}$ and $A$. To this end, we proceed in three steps:

Step 1. We first calculate $U_{M B}$, where $U_{M B}$ is the representation of $U$ in the magic basis (3):

$$
U_{M B} \equiv Q^{\dagger} U Q=O_{1} F O_{2} \text {. }
$$

We then define vectors $\left|v_{i}\right\rangle$ as

$$
\left|v_{i}\right\rangle \equiv O_{2}^{-1}\left|e_{i}\right\rangle \quad \text { for } \quad i=1, \ldots, 4 .
$$

The vectors (9) are also the eigenvectors of $U_{M B}^{T} U_{M B}$ with corresponding eigenvalues $\mu_{i} \equiv \lambda_{i}^{2}$ :

$$
U_{M B}^{T} U_{M B}\left|v_{i}\right\rangle=O_{2}^{T} F^{2} O_{2}\left|v_{i}\right\rangle=O_{2}^{T} F^{2}\left|e_{i}\right\rangle=\lambda_{i}^{2} O_{2}^{T}\left|e_{i}\right\rangle=\mu_{i}\left|v_{i}\right\rangle .
$$

Thus by calculating the eigenvalues and eigenvectors of $U_{M B}^{T} U_{M B}$, which is a known matrix, we directly obtain the matrix $\mathrm{O}_{2}$ from equation (9) as

$$
\mathrm{O}_{2}=\left(\left|v_{1}\right\rangle \cdots\left|v_{4}\right\rangle\right)^{T}
$$

and hence, using equation (5),

$$
K_{2}=Q\left(\left|v_{1}\right\rangle \ldots\left|v_{4}\right\rangle\right)^{T} Q^{\dagger} .
$$

In the above we are free to assign the eigenvectors of $U_{M B}^{T} U_{M B}$ in some chosen order to the vectors $\left|v_{i}\right\rangle$, with the restriction that since $O_{2} \in S O(4)$ it should hold that $\operatorname{det} O_{2}=1$.

Step 2. Using the eigenvalues $\lambda_{i}^{2}$ of $U_{M B}^{T} U_{M B}$ found in step 1, we calculate the arguments $\alpha, \beta$ and $\gamma$ of the matrix $A$ (equation (1) $)^{5}$ using (7),

$$
\begin{aligned}
& \alpha=\frac{-\mathrm{i}}{4} \log \left(\lambda_{1} \lambda_{2} \lambda_{3}^{-1} \lambda_{4}^{-1}\right)+\frac{1}{2} k_{1} \pi \\
& \beta=\frac{-\mathrm{i}}{4} \log \left(\lambda_{1}^{-1} \lambda_{2} \lambda_{3}^{-1} \lambda_{4}\right)+\frac{1}{2} k_{2} \pi \\
& \gamma=\frac{-\mathrm{i}}{4} \log \left(\lambda_{1} \lambda_{2}^{-1} \lambda_{3}^{-1} \lambda_{4}\right)+\frac{1}{2} k_{3} \pi,
\end{aligned}
$$

where $k_{1}, k_{2}, k_{3} \in \mathbb{Z}$.

Step 3. Finally, the matrix $O_{1}$ is calculated from

$$
O_{1}=O_{1} F O_{2} O_{2}^{T} F^{*}=U_{M B} O_{2}^{T} F^{*}
$$

and hence

$$
\begin{aligned}
K_{1} & =Q U_{M B} O_{2}^{T} F^{*} Q^{\dagger} \\
& =U\left(Q Q^{T}\right) K_{2}^{T} A^{*}\left(Q Q^{T}\right)^{*} .
\end{aligned}
$$

${ }^{4}$ Equations (7) were also given in [4]. In the following, we go beyond this result and derive explicit expressions for $\alpha, \beta$ and $\gamma$.

5 The values $\alpha, \beta$ and $\gamma$ given here (each of which depends on $\lambda_{1}-\lambda_{4}$ ) are not unique and other expressions are equally valid, e.g. $\alpha=\frac{-\mathrm{i}}{2} \log \left(\lambda_{1} \lambda_{2}\right)+\frac{1}{2} k_{1} \pi$. 
There are two degrees of freedom in this decomposition procedure. First, there are different ways of identifying the eigenvectors of $U_{M B}^{T} U_{M B}$ with the vectors $\left|v_{i}\right\rangle$. Secondly, there is some freedom in choosing the arguments $\alpha, \beta$ and $\gamma$ of the operation $A$. In general, different choices lead to different matrices $K_{1}, A$ and $K_{2}$, and the $K_{1} A K_{2}$-decomposition of $U$ is not unique.

At this point it is useful to compare the procedure for obtaining a $K_{1} A K_{2}$-decomposition described above to that proposed by Kraus and Cirac (KC) [4]. There are two main differences between the two: (1) using KC's method the matrix $K_{2}$ is given in the form $S U(2) \times S U(2)$ and involves three unknowns (Euler angles), and (2) KC's method leads to fixed values of the $\lambda_{i}$ 's (instead of freedom of choice of the sign of each $\lambda_{i}$ ). Which of the two methods is more convenient to use depends on the goal of the calculation to be made. If this goal is to (only) find a $K_{1} A K_{2}$ decomposition of a given matrix $U$, then having $K_{2}$ in $S U(2) \times S U(2)$ form and the $\lambda_{i}$ 's fixed may be advantageous, if it leads to less equations to be solved. If, on the other hand, the goal of the calculation is to first find a $K_{1} A K_{2}$ decomposition of $U$ and then decompose each of the matrices $K_{1}, A$ and $K_{2}$ in terms of single-qubit rotations and a given tunable two-qubit interaction $U_{\text {int }}(t)$ (as it is in this paper), the freedom of assigning the vectors $\left|v_{i}\right\rangle$ and choosing the sign of the $\lambda_{i}$ 's that our method entails is advantageous, since it allows us to choose these parameters in such a way that the ensuing decomposition into single-qubit rotations and $U_{\mathrm{int}}(t)$ is straightforward.

\subsection{Maximally entangling gates $U \in S U(4)$}

The procedure described in the previous subsection can be simplified if $U$ is a quantum gate which maximally entangles a suitably chosen separable basis, which we refer to as a 'maximally entangling operator' or a 'quantum gate with maximally entangling capacity' (not to be confused with quantum gates which 'only' maximally entangle a single separable quantum state instead of a whole basis). For this special class of two-qubit operations the following proposition holds:

Proposition 1. Any transformation $M \in S U(4)$ from the standard basis to a maximallyentangled basis can be expressed in the following way:

$$
M=K_{1} \mathrm{e}^{\frac{1}{4} \mathrm{i} \pi\left(\sigma_{x} \otimes \sigma_{x}+\epsilon \sigma_{z} \otimes \sigma_{z}\right)} R_{x}^{(1)}(\mu) R_{x}^{(2)}(\nu) R_{y}^{(1)}(\xi) R_{y}^{(2)}(\zeta) R_{z}^{(1)}(\eta) R_{z}^{(2)}(\omega),
$$

where $\epsilon, \mu, \nu, \xi, \zeta, \eta$ and $\omega$ satisfy one of the following three conditions:

(1) $\epsilon=0, \quad \xi, \zeta \in\{0, \pm \pi\}, \quad \mu, \nu, \eta, \omega \in[0,2 \pi)$

(2) $0<\epsilon<1, \quad \mu, \nu, \xi, \zeta \in\{0, \pm \pi\}, \quad \eta, \omega \in[0,2 \pi)$

(3) $\epsilon=1, \quad \mu, v \in\{0, \pm \pi\}, \quad \xi, \zeta, \eta, \omega \in[0,2 \pi)$.

Proof. It has recently been shown [9] that any maximally entangling operator is of the form $A(\epsilon) \equiv \exp \left[(1 / 4) i \pi\left(\sigma_{x} \otimes \sigma_{x}+\epsilon \sigma_{z} \otimes \sigma_{z}\right)\right]$ (with $\epsilon \in[0,1]$ and modulo permutations in $\left.\sigma_{x}, \sigma_{y}, \sigma_{z}\right)$. We are thus left with the question of which rotations may be performed on the standard basis before applying $A(\epsilon)$, such that $A(\epsilon)$ acts as a maximally entangling operation. Since rotations around the $z$-axis only multiply each quantum state by a phasefactor, they are always allowed. It is then necessary and sufficient to investigate for which values of $\epsilon, \mu, \nu, \xi$ and $\zeta$ the transformation $T \equiv A(\epsilon) R_{x}^{(1)}(\mu) R_{x}^{(2)}(\nu) R_{y}^{(1)}(\xi) R_{y}^{(2)}(\zeta)$ corresponds to a maximally entangling operation. These values can be found in a straightforward way by determining for which values of $\epsilon, \mu, v, \xi$ and $\zeta$ the concurrence [10] equals 1 for each of the 
four column vectors $\left|w_{i}\right\rangle$. The calculation is given in the appendix and leads to the solution equation (14).

Note that in order to transform from the standard basis to a specific maximally entangled basis the conditions on the angles in equation (14) are in general more restrictive. For example, to transform to the Bell basis we require $\mu, v, \xi, \zeta \in\{0, \pm \pi\}, \eta, \omega \in[0,2 \pi)$ and $\alpha_{i}, \beta_{i}, \gamma_{i} \in[0, \pm \pi / 2, \pm \pi]$ for all values of $\epsilon$.

For gates $U \in S U(4)$ which maximally entangle the standard basis, step 2 in the decomposition procedure from the previous subsection can now be simplified: using proposition 1 we choose $\lambda_{1}-\lambda_{4}$ such that $\beta=0$ and $\alpha=\pi / 4$. For each of the possible assignments we then calculate $\gamma$ (and thus the value of $\epsilon$ in equation (13)) from equation $(11 c)$.

Having found the decomposition of $U$ into the matrices $K_{1}, K_{2}$ and $A$, we now proceed to develop a systematic scheme to calculate the implementation of each of these matrices using a sequence of single-qubit rotations (for $K_{1}$ and $K_{2}$ ) plus two-qubit interactions $U_{\text {int }}(t)$ of a given kind (for $A$ ). This is the topic of the next subsection.

\subsection{Decomposing $K_{1}, K_{2}$ and $A$ into $U_{\mathrm{int}}(t)$ and single-qubit rotations}

We first consider gates $U \in S U(4)$ with maximally entangling capacity, as defined in the previous subsection.

2.3.1. Decomposition of $\mathrm{K}_{2}$ into single-qubit rotations. For maximally entangling twoqubit gates, a decomposition of the matrix $K_{2}$ into single-qubit rotations can be found in a straightforward way by comparing the matrix $K_{2}$ obtained from (10) to the sequence of rotations on the right-hand side of equation (13), using the appropriate conditions on the angles (14) that correspond to the value of $\epsilon=4 \gamma / \pi$ calculated from equation (11c). The resulting decomposition of $K_{2}$ is not unique, as can be seen from (14) where different sequences of single-qubit rotations each correspond to a correct decomposition of $K_{2}$.

2.3.2. Decomposition of $K_{1}$ into single-qubit rotations. $K_{1}$ can be translated into single-qubit rotations using the Euler decomposition theorem [11], which states that every $K \in S U(2) \otimes S U(2)$ can be written as

$$
K=\prod_{j=1,2} R_{z}^{(j)}\left(\alpha_{j}\right) R_{y}^{(j)}\left(\beta_{j}\right) R_{z}^{(j)}\left(\gamma_{j}\right)
$$

Here $R_{z}^{(j)}\left(\alpha_{j}\right)$ represents a rotation of qubit $j$ over $\alpha_{j}$ around the $z$-axis and $\alpha_{j}, \beta_{j}, \gamma_{j} \in$ $[0,2 \pi)$. By substituting $K_{1}$ calculated from equation (12) into the left-hand side of equation (15) and solving the resulting set of equations, we directly obtain the values of the angles $\alpha_{i}, \beta_{i}$ and $\gamma_{i}$. It is clear that, as for $K_{2}$, the decomposition of $K_{1}$ is not unique and that different sequences of rotations all yield a correct decomposition of $K_{1}$.

2.3.3. Decomposition of $A$ into $U_{\text {int }}(t)$ and single-qubit rotations. Our next goal is to decompose the matrix $A$ (equation (1) with the values of $\alpha, \beta$ and $\gamma$ obtained from equation (11)) into a sequence of two-qubit interactions $U_{\text {int }}(t)$ and single-qubit rotations such that the smallest number of $U_{\text {int }}(t)$ is used. To this end, we first examine the entangling capacity of the given two-qubit gate $U_{\text {int }}(t)$ as a function of $t$, i.e. we investigate how many applications of $U_{\text {int }}(t)$ are needed to transform the standard basis into a maximally entangled basis for a given $t$. For tunable two-qubit gates $U_{\text {int }}(t)$ there exists in general a value of 
$t$, say $t^{*}$, for which this minimum number of applications is small. The value of $t^{*}$ is obtained by calculating a measure of bipartite entanglement, such as the concurrence [10], for $U_{\text {int }}(t)$ applied to the standard basis vectors. In many cases $t^{*}$ can be determined directly from inspection of $U_{\text {int }}(t)$ (for $U_{\text {int }}(t)$ written in the standard basis). For example, if $U_{\text {int }}(t)$ is the (SWAP) ${ }^{\alpha}$-gate (see section 3, equation (17)), $t^{*}$ is determined by $\alpha$. In this case two applications of (SWAP) ${ }^{1 / 2}$ (plus a single-qubit rotation in between) are sufficient to implement $U[12,13]^{6}$ since (SWAP) $)^{1 / 2}$ maximally entangles two out of four standard basis states (and affects the other two basis states only trivially, by multiplication with a phase factor). Therefore $t^{*}=\pi /(2 \hbar J)$ (for constant $J$, see equation (16)).

Once a sequence of operations, say $\tilde{A}$, that transforms the standard basis into a maximally entangled basis and contains the smallest possible number of $U_{\text {int }}$ (plus single-qubit rotations) has been found, $A$ and $\tilde{A}$ differ by local operations only. The latter can be calculated in the same way as for $K_{1}$, by using the Euler decomposition (15) of $A \tilde{A}^{-1}$.

For two-qubit entangling gates $U \in S U(4)$ that do not have maximally entangling capacity, the decomposition procedure of $K_{1}, A$ and $K_{2}$ into $U_{\text {int }}(t)$ and single-qubit rotations is the same as for maximally entangling gates described above-except that the matrix $K_{2}$ now also has to be decomposed by using the Euler decomposition (15), since proposition 1 does not apply.

\subsection{Optimization}

By joining together the individual decompositions of $K_{1}, A$ and $K_{2}$ obtained via the method described in the previous subsection, we have obtained a decomposition of $U=K_{1} A K_{2}$ which consists of the smallest number of two-qubit interactions (plus a number of singlequbit rotations). This optimal decomposition is obtained by first optimizing the number of two-qubit gates and subsequently optimizing the number of single-qubit gates. The total number of rotations in this decomposition can often be reduced by using one or both of the following:

(i) Commutation relations between $U_{\text {int }}(t)$ (or $\tilde{A}$, as defined in the previous subsection) and rotations,

(ii) Euler's theorem: writing rotations $R_{m_{i}}^{(j)}(\phi)$ as $^{7} R_{n_{i}}^{(j)}\left(\alpha_{1}\right) R_{p_{i}}^{(j)}(\phi) R_{n_{i}}^{(j)}\left(\alpha_{2}\right)$ with $n_{i} \neq p_{i}$, both $n_{i}$ and $p_{i}$ orthogonal to $m_{i}$, and $\alpha_{1}, \alpha_{2} \in\{ \pm \pi / 2\}$ (and using (i) again).

Once both of these do not lead to a further reduction of the number of single-qubit rotations, the minimum number of rotations has been found.

In practice, depending on experimental conditions, it may only be possible (or be easier) to implement rotations around certain axes and therefore be necessary to e.g. translate rotations around the $z$-axis into a sequence of rotations around axes in the $(x, y)$-plane. By implementing specific requirements such as these and using commutation relations to minimize the number of rotations we obtain the implementation of $U$ which requires the smallest number of operations from a given library of single- and two-qubit operations.

6 For non-tunable entangling gates $U_{\text {int }}$ the task of analytically determining the number of operations that is required for implementation of a desired gate $U$ is in general more difficult. One can, however, always analytically find a closed-from solution for the required quantum circuit and an upper bound for the required number of applications of $U_{\text {int }}$ or use numerical techniques to minimize the required number of $U_{\text {int }}$ [12].

7 This is always possible and follows from Euler's theorem [11]. 


\section{Illustration of the decomposition protocol}

In this section we illustrate the protocol developed in the previous section for two types of interaction $U_{\text {int }}(t)$ : Heisenberg exchange interaction and quantum inductive coupling. The first one is described by the Hamiltonian

$$
\mathcal{H}_{E X}(t)=(1 / 4) \hbar^{2} J(t) \vec{\sigma}^{(1)} \cdot \vec{\sigma}^{(2)},
$$

with $J(t)$ the time-dependent (tunable) exchange energy. This interaction corresponds to the $(\mathrm{SWAP})^{\alpha}$-gate: $(\mathrm{SWAP})^{\alpha} \equiv U_{E X}(t)=\exp \left[-(\mathrm{i} / \hbar) \int_{0}^{t} \mathcal{H}_{E X}(\tau) \mathrm{d} \tau\right]$ with

$$
\alpha(t) \equiv-\frac{\hbar}{\pi} \int_{0}^{t} J(\tau) \mathrm{d} \tau
$$

or, equivalently,

$$
(\mathrm{SWAP})^{\alpha}=\mathrm{e}^{-\frac{\alpha}{4} \mathrm{i} \pi}\left(\begin{array}{cccc}
\mathrm{e}^{\frac{\alpha}{2} \mathrm{i} \pi} & 0 & 0 & 0 \\
0 & \cos \left(\frac{\alpha}{2} \pi\right) & \mathrm{i} \sin \left(\frac{\alpha}{2} \pi\right) & 0 \\
0 & \mathrm{i} \sin \left(\frac{\alpha}{2} \pi\right) & \cos \left(\frac{\alpha}{2} \pi\right) & 0 \\
0 & 0 & 0 & \mathrm{e}^{\frac{\alpha}{2} \mathrm{i} \pi}
\end{array}\right) .
$$

The second type of interaction we use to illustrate our protocol is a tunable coupling between two magnetic fluxes (see section 4 for a description of a physical realization using the so-called flux qubits). The Hamiltonian corresponding to this type of interaction is given by [14]

$$
\mathcal{H}_{\text {ind }}(t) \equiv-\frac{g_{+}(t)}{4}\left(\sigma_{x} \otimes \sigma_{x}-\sigma_{y} \otimes \sigma_{y}\right)-\frac{g_{-}(t)}{4}\left(\sigma_{x} \otimes \sigma_{x}+\sigma_{y} \otimes \sigma_{y}\right),
$$

$\gamma_{ \pm}(t) \equiv \frac{1}{\hbar} \int_{0}^{t} g_{ \pm}(\tau) \mathrm{d} \tau$, where $g_{ \pm}(t)$ are tunable system parameters. The Hamiltonian (18) corresponds to the so-called B-gate [3]: $B\left(\gamma_{+}, \gamma_{-}\right) \equiv U_{\text {int }}(t)=\exp \left[-\frac{\mathrm{i}}{\hbar} \int_{0}^{t} \mathcal{H}_{\text {int }}(\tau) \mathrm{d} \tau\right]$ or, equivalently,

$$
B\left(\gamma_{+}, \gamma_{-}\right)=\left(\begin{array}{cccc}
\cos \left(\frac{\gamma_{+}}{2}\right) & 0 & 0 & \mathrm{i} \sin \left(\frac{\gamma_{+}}{2}\right) \\
0 & \cos \left(\frac{\gamma_{-}}{2}\right) & \mathrm{i} \sin \left(\frac{\gamma_{-}}{2}\right) & 0 \\
0 & \mathrm{i} \sin \left(\frac{\gamma_{-}}{2}\right) & \cos \left(\frac{\gamma_{-}}{2}\right) & 0 \\
\mathrm{i} \sin \left(\frac{\gamma_{+}}{2}\right) & 0 & 0 & \cos \left(\frac{\gamma_{+}}{2}\right)
\end{array}\right)
$$

with $\gamma_{ \pm} \in[0,2 \pi)$. Note that equation (19) for $\gamma_{+}, \gamma_{-}= \pm \pi / 2$ maximally entangles the entire standard basis, whereas the exchange interaction $\sqrt{\mathrm{SWAP}}$ only produces maximal entanglement for two out of the four states in the standard basis. For both $\mathcal{H}_{E X}(t)$ and $\mathcal{H}_{\text {ind }}(t)$ we now calculate the optimal (shortest-sequence) implementation of three elementary quantum operations: the transformation from standard basis to Bell basis, the CNOT-gate and the quantum Fourier transform. The former two are maximally entangling operations, but the latter, as shown below, is not.

\subsection{Transformation from standard to Bell basis}

A transformation from the standard basis to the Bell basis is by definition a maximally entangling operation. It has been shown that when using the Heisenberg exchange interaction (The (SWAP) ${ }^{\alpha}$-gate, equation (17)) the shortest sequence of operations that transforms the 
standard basis into a Bell basis is given by [7, 13]

$$
\begin{aligned}
M_{n_{\phi}}^{(j)} & \equiv \sqrt{\operatorname{SWAP}} R_{n_{\phi}}^{(j)}(\pi) \sqrt{\operatorname{SWAP}} \\
& \stackrel{j=1}{=} \frac{\mathrm{e}^{-\mathrm{i} \frac{\pi}{4}}}{\sqrt{2}}\left(\begin{array}{cccc}
0 & \mathrm{e}^{-\mathrm{i} \phi} & -\mathrm{i} \mathrm{e}^{-\mathrm{i} \phi} & 0 \\
\mathrm{e}^{\mathrm{i} \phi} & 0 & 0 & -\mathrm{ie}^{-\mathrm{i} \phi} \\
-\mathrm{i} \mathrm{e}^{\mathrm{i} \phi} & 0 & 0 & \mathrm{e}^{-\mathrm{i} \phi} \\
0 & -\mathrm{ie}^{\mathrm{i} \phi} & \mathrm{e}^{\mathrm{i} \phi} & 0
\end{array}\right),
\end{aligned}
$$

where $j=1,2$ labels the qubit and $R_{n_{\phi}}^{(j)}(\pi)$ represents a rotation of qubit $j$ around an arbitrary axis $n_{\phi} \equiv(\cos \phi, \sin \phi, 0)$ in the $(x, y)$-plane. The analogous transformation for the quantum inductive coupling consists of a single application ${ }^{8}$ of the $B$-gate (equation (19)) with $\gamma_{+}, \gamma_{-}= \pm \frac{\pi}{2}$. In order to find the transformation from the standard basis to the 'standard Bell basis' $\{(1 / \sqrt{2})(|\uparrow \uparrow\rangle \pm|\downarrow \downarrow\rangle),(1 / \sqrt{2})(|\uparrow \downarrow\rangle \pm|\downarrow \uparrow\rangle)\}$, we need to decompose the matrix

$$
U^{\mathrm{st} \rightarrow \text { Bell }}=\frac{1}{\sqrt{2}}\left(\begin{array}{cccc}
1 & 0 & 0 & 1 \\
0 & 1 & 1 & 0 \\
0 & 1 & -1 & 0 \\
1 & 0 & 0 & -1
\end{array}\right)
$$

(or a permutation of (21) in which the columns are interchanged). Using the protocol outlined in the previous section, we first calculate the matrices $K_{1}, A$, and $K_{2}$ and then decompose these matrices into the shortest sequence of $U_{\text {int }}=(\text { SWAP })^{\alpha}$ or $U_{\text {int }}=B$ plus single-qubit rotations. Starting with step 1 in section 2.1 , we find that

$$
U_{M B}^{\mathrm{st} \rightarrow \text { Bell }}=\frac{1}{\sqrt{2}}\left(\begin{array}{cccc}
1 & 0 & 0 & \mathrm{i} \\
0 & 1 & -\mathrm{i} & 0 \\
0 & \mathrm{i} & -1 & 0 \\
-\mathrm{i} & 0 & 0 & -1
\end{array}\right)
$$

and the eigenvalues and the corresponding eigenvectors of $\left(U_{M B}^{\mathrm{st} \rightarrow \text { Bell }^{\mathrm{T}}} U_{M B}^{\mathrm{st} \rightarrow}\right.$ Bell $)$ are given by

$$
\begin{array}{ll}
\mu_{1}=\mu_{2}=\mathrm{i}, \quad\left|a_{1}\right\rangle=\frac{1}{\sqrt{2}}\left(\begin{array}{l}
1 \\
0 \\
0 \\
1
\end{array}\right), \quad\left|a_{2}\right\rangle=\frac{1}{\sqrt{2}}\left(\begin{array}{c}
0 \\
-1 \\
1 \\
0
\end{array}\right) \\
\mu_{3}=\mu_{4}=-\mathrm{i}, \quad\left|a_{3}\right\rangle=\frac{1}{\sqrt{2}}\left(\begin{array}{l}
0 \\
1 \\
1 \\
0
\end{array}\right) \quad\left|a_{4}\right\rangle=\frac{1}{\sqrt{2}}\left(\begin{array}{c}
1 \\
0 \\
0 \\
-1
\end{array}\right) .
\end{array}
$$

We now choose $\left|v_{i}\right\rangle \equiv\left|a_{i}\right\rangle, \forall i=1, \ldots, 4$, and calculate the matrix $K_{2}$ using (10):

$$
K_{2}^{\mathrm{st} \rightarrow \text { Bell }}=\frac{1}{\sqrt{2}}\left(\begin{array}{cccc}
0 & 0 & 0 & 1+\mathrm{i} \\
0 & 0 & -1-\mathrm{i} & 0 \\
0 & -1+\mathrm{i} & 0 & 0 \\
1-\mathrm{i} & 0 & 0 & 0
\end{array}\right) .
$$

\footnotetext{
8 If a single application of $U_{\text {int }}$ is sufficient to implement $U, U$ and $U_{\text {int }}$ are locally equivalent. See also [15] in which
} entanglement invariants are presented that can be used to verify local equivalence of two-qubit gates. 
Next, we use the eigenvalues $\lambda_{i} \equiv \sqrt{\mu_{i}}, i=1, \ldots, 4$ (equation (22)) to calculate the matrix $A$ from equations (11) and (1). Choosing $\lambda_{1}=\sqrt{\mathrm{i}}, \lambda_{2}=-\sqrt{\mathrm{i}}, \lambda_{3}=\sqrt{-\mathrm{i}}, \lambda_{4}=-\sqrt{-\mathrm{i}}$ we find the solution $\alpha=\pi / 4, \beta=\gamma=0$ and hence

$$
A^{\mathrm{st} \rightarrow \text { Bell }}=\frac{1}{\sqrt{2}}\left(\begin{array}{cccc}
1 & 0 & 0 & \mathrm{i} \\
0 & 1 & \mathrm{i} & 0 \\
0 & \mathrm{i} & 1 & 0 \\
\mathrm{i} & 0 & 0 & 1
\end{array}\right)
$$

Finally, we obtain $K_{1}$ from equation (12):

$$
K_{1}^{\mathrm{st} \rightarrow \text { Bell }}=\frac{1}{\sqrt{2}}\left(\begin{array}{cccc}
1-\mathrm{i} & 0 & 0 & 0 \\
0 & -1+\mathrm{i} & 0 & 0 \\
0 & 0 & -1-\mathrm{i} & 0 \\
0 & 0 & 0 & 1+\mathrm{i}
\end{array}\right) .
$$

We now decompose each of the matrices $K_{2}^{\mathrm{st} \rightarrow \text { Bell }}, A^{\mathrm{st} \rightarrow \text { Bell }}$ and $K_{1}^{\mathrm{st} \rightarrow \text { Bell }}$ (equations (23)-(25)) into a sequence of single-qubit rotations and (SWAP) ${ }^{\alpha}$-gates, using the procedure described in section 2.3. Starting with $K_{2}^{\text {st } \rightarrow \text { Bell }}$ and comparing the matrix (23) to the right-hand side of equation (13) for condition (14a), we find

$$
K_{2}^{\mathrm{st} \rightarrow \mathrm{Bell}}=R_{y}^{(1)}(\pi) R_{y}^{(2)}(\pi) R_{z}^{(1)}\left(\frac{\pi}{2}\right)
$$

By comparing the matrices in equations (24) and (20), we directly obtain a decomposition of $A^{\text {st } \rightarrow \text { Bell }}$, since for $n_{\phi}=x$ and $j=1$ the two matrices only differ by a spin flip of the first qubit (which exchanges the first and third rows as well as the second and fourth rows). Hence we find, disregarding a global phasefactor,

$$
A^{\mathrm{st} \rightarrow \text { Bell }}=R_{x}^{(1)}(\pi) \sqrt{\operatorname{SWAP}} R_{x}^{(1)}(\pi) \sqrt{\mathrm{SWAP}} .
$$

This decomposition of $A^{\mathrm{st} \rightarrow \text { Bell }}$ is also obtained by following the decomposition procedure described in section 2.3 , by first calculating the entangling capacity of (SWAP) ${ }^{\alpha} \forall \alpha$. Finally, the decomposition of the matrix $K_{1}^{\text {st } \rightarrow \text { Bell }} \in S U(2) \otimes S U(2)$ is obtained by using equation (15), and we find

$$
K_{1}^{\mathrm{st} \rightarrow \text { Bell }}=R_{z}^{(1)}\left(-\frac{\pi}{2}\right) R_{z}^{(2)}(\pi)
$$

The total decomposition of $U^{\text {st } \rightarrow \text { Bell }}$ in terms of $\sqrt{\text { SWAP }}$-operations then becomes

$$
\begin{aligned}
U^{\mathrm{st} \rightarrow \text { Bell }} & =K_{1}^{\mathrm{st} \rightarrow \text { Bell }} A^{\mathrm{st} \rightarrow \text { Bell }} K_{2}^{\mathrm{st} \rightarrow \text { Bell }} \\
& =R_{z}^{(1)}\left(-\frac{\pi}{2}\right) R_{z}^{(2)}(\pi) R_{x}^{(1)}(\pi) \sqrt{\operatorname{SWAP}} R_{x}^{(1)}(\pi) \sqrt{\operatorname{SWAP}} R_{y}^{(1)}(\pi) R_{y}^{(2)}(\pi) R_{z}^{(1)}\left(\frac{\pi}{2}\right) \\
& =R_{z}^{(1)}\left(-\frac{\pi}{2}\right) \sqrt{\operatorname{SWAP}} R_{x}^{(1)}(\pi) \sqrt{\operatorname{SWAP}} R_{y}^{(1)}(\pi) R_{z}^{(1)}\left(\frac{\pi}{2}\right) .
\end{aligned}
$$

In the last step of equation (29) we have used the relation $\sqrt{\operatorname{SWAP}} R_{x}^{(1)}(\pi) \sqrt{\operatorname{SWAP}} R_{y}^{(2)}(\pi)=$ $R_{z}^{(2)}(\pi) R_{x}^{(1)}(\pi) \sqrt{\mathrm{SWAP}} R_{x}^{(1)}(\pi) \sqrt{\mathrm{SWAP}}$. Equation (29) is the shortest sequence of operations that can be used to implement $U^{\text {st } \rightarrow \text { Bell }}$ using Heisenberg exchange interaction and single-qubit rotations. Repeating the same procedure for other transformations from the standard basis to 
the Bell basis (obtained by permutations of the columns of equation (21)) we find the following possible optimal decompositions of $U^{\mathrm{st} \rightarrow \text { Bell }}$ :

$$
U_{(\mathrm{SWAP})^{\alpha}}^{\mathrm{st} \rightarrow \mathrm{Beel}}=\left\{\begin{array}{l}
R_{z}^{(1)}\left(\sigma \frac{\pi}{2}\right) M_{x}^{(i)} R_{z}^{(1)}\left( \pm \frac{\pi}{2}\right) \\
R_{z}^{(1)}\left(-\sigma \frac{\pi}{2}\right) R_{x}^{(1)}(\pi) M_{x}^{(i)} R_{z}^{(1)}\left( \pm \frac{\pi}{2}\right) \\
R_{z}^{(1)}\left(\sigma \frac{\pi}{2}\right) R_{y}^{(1)}(\pi) M_{x}^{(i)} R_{z}^{(1)}\left( \pm \frac{\pi}{2}\right) \\
R_{z}^{(1)}\left(-\sigma \frac{\pi}{2}\right) M_{x}^{(i)} R_{x, y}^{(1)}(\pi) R_{z}^{(1)}\left( \pm \frac{\pi}{2}\right)
\end{array}\right.
$$

with $(\mathrm{i}, \sigma) \in\{(1,1),(2,-1)\}^{9}$.

The analogue of equation (30) for quantum induced coupling (the B-gate, equation (19)) is given by:

$$
U_{\mathrm{B}}^{\mathrm{st} \rightarrow \text { Bell }}=\left\{\begin{array}{l}
R_{z}^{(1)}\left(-\sigma \frac{\pi}{2}\right) B\left(\sigma \frac{\pi}{2}, \sigma \frac{\pi}{2}\right) R_{z}^{(1)}\left( \pm \frac{\pi}{2}\right) \\
R_{z}^{(1)}\left(\sigma \frac{\pi}{2}\right) R_{x}^{(1)}(\pi) B\left(\sigma \frac{\pi}{2}, \sigma \frac{\pi}{2}\right) R_{z}^{(1)}\left( \pm \frac{\pi}{2}\right) \\
R_{z}^{(1)}\left(-\sigma \frac{\pi}{2}\right) R_{x}^{(1)}(\pi) B\left(\sigma \frac{\pi}{2}, \sigma \frac{\pi}{2}\right) R_{x, y}^{(1)}(\pi) R_{z}^{(1)}\left( \pm \frac{\pi}{2}\right) \\
R_{z}^{(1)}\left(\sigma \frac{\pi}{2}\right) R_{y}^{(1)}(\pi) B\left(\sigma \frac{\pi}{2}, \sigma \frac{\pi}{2}\right) R_{x}^{(1)}(\pi) R_{z}^{(1)}\left( \pm \frac{\pi}{2}\right) \\
R_{z}^{(1)}\left(\sigma \frac{\pi}{2}\right) B\left(\sigma \frac{\pi}{2}, \sigma \frac{\pi}{2}\right) R_{x}^{(1)}(\pi) R_{z}^{(1)}\left( \pm \frac{\pi}{2}\right)
\end{array}\right.
$$

with $\sigma= \pm 1$. Note that equations (30) and (31) involve six rotations more than the decomposition of $M_{n_{\phi}}$, equation (20). From a practical point of view, it is thus more efficient to implement a transformation from the standard basis to a Bell basis with complex coefficients than to the 'standard Bell basis' when either $U_{\text {int }}=(\mathrm{SWAP})^{\alpha}$ or $U_{\text {int }}=B$ is used.

To conclude this section, we also give the decomposition of two alternative matrices $A^{\text {st } \rightarrow \text { Bell }}$. The first one is obtained by using the $K_{1} A K_{2}-$ decomposition procedure of Kraus and Cirac [4] and reads

$$
A_{\text {alt1 }}^{\mathrm{st} \rightarrow \text { Bell }}=\left(\begin{array}{cccc}
0 & 0 & 0 & 1 \\
0 & 0 & -\mathrm{i} & 0 \\
0 & -\mathrm{i} & 0 & 0 \\
1 & 0 & 0 & 0
\end{array}\right) .
$$

$A_{\text {alt1 }}^{\text {st } \rightarrow \text { Bell }}$ does not maximally entangle the standard basis. In order to find its decomposition, we first calculate an unentangled basis for which $A_{\text {alt } 1}^{\text {st } \rightarrow \text { Bell }}$ does act as a maximal entangler. To this end, we note from the matrix (32) that the latter basis must be obtained from the standard basis by creating a superposition of both the first and the second qubit, since equation (32) couples $|\uparrow \uparrow\rangle$ to $|\downarrow \downarrow\rangle$ and $|\uparrow \downarrow\rangle$ to $|\downarrow \uparrow\rangle$. Mathematically, a possible choice of rotations is

$$
\left(\begin{array}{cccc}
1 & -1 & -1 & 1 \\
1 & 1 & -1 & -1 \\
-1 & 1 & -1 & 1 \\
-1 & -1 & -1 & -1
\end{array}\right)
$$

which translates into (using Euler's decomposition theorem) $R_{y}^{(1)}\left(\frac{\pi}{2}\right) R_{z}^{(1)}(\pi) R_{y}^{(2)}\left(-\frac{\pi}{2}\right)$. Thus $A_{\text {alt } 1}^{\mathrm{st} \rightarrow \text { Bell }} R_{y}^{(1)}\left(\frac{\pi}{2}\right) R_{z}^{(1)}(\pi) R_{y}^{(2)}\left(-\frac{\pi}{2}\right)=$ (basis rotation) $\times \sqrt{\operatorname{SWAP}} R_{x}^{(1)}(\pi) \sqrt{\mathrm{SWAP}} \equiv$ (basis rotation) $\times M_{x}^{(1)}$. The remaining basis rotation is easily found by using Euler's theorem and the full decomposition then reads

$$
A_{\text {alt1 }}^{\mathrm{st} \rightarrow \text { Bell }}=R_{y}^{(1)}\left(\frac{\pi}{2}\right) R_{y}^{(2)}\left(\frac{\pi}{2}\right) M_{x}^{(1)} R_{z}^{(1)}(\pi) R_{y}^{(1)}\left(-\frac{\pi}{2}\right) R_{y}^{(2)}\left(\frac{\pi}{2}\right) .
$$

9 If only rotations in the $(x, y)$-plane are available, $z$-rotations can be implemented using $R_{z}^{(1)}(\phi)=$ $R_{y}^{(1)}\left(-\frac{\pi}{2}\right) R_{x}^{(1)}(\phi) R_{y}^{(1)}\left(\frac{\pi}{2}\right)$ or $R_{z}^{(1)}(\phi)=R_{x}^{(1)}\left(\frac{\pi}{2}\right) R_{y}^{(1)}(\phi) R_{x}^{(1)}\left(-\frac{\pi}{2}\right)$. 
As a second example of an alternative matrix $A_{\mathrm{alt2}}^{\mathrm{st} \rightarrow \text { Bell }}$, we consider the matrix obtained by using a different choice of $\lambda_{i}$ 's, namely $\lambda_{1}=\sqrt{-i}, \lambda_{2}=-\sqrt{i}, \lambda_{3}=\sqrt{i}$ and $\lambda_{4}=-\sqrt{-i}$. For this choice the matrix $A$ (equation (1)) becomes

$$
A_{\text {alt2 }}^{\text {st } \rightarrow \text { Bell }}=\left(\begin{array}{cccc}
1 & 0 & 0 & 0 \\
0 & -\mathrm{i} & 0 & 0 \\
0 & 0 & -\mathrm{i} & 0 \\
0 & 0 & 0 & 1
\end{array}\right) .
$$

$A_{\mathrm{alt2}}^{\mathrm{st} \rightarrow}$ Bell acts as a maximal entangler on the same unentangled basis as $A_{\text {alt1 }}^{\mathrm{st} \rightarrow \text { Bell }}$ (see above), so that $A_{\text {alt2 }}^{\text {st } \rightarrow \text { Bell }} R_{y}^{(1)}\left(\frac{\pi}{2}\right) R_{z}^{(1)}(\pi) R_{y}^{(2)}\left(-\frac{\pi}{2}\right)=$ (basis rotation) $\times M_{x}^{(1)}$. The remaining basis rotation is again found by decomposing $A_{\text {alt2 }}^{\mathrm{st} \rightarrow}$ Bell $R_{y}^{(1)}\left(\frac{\pi}{2}\right) R_{z}^{(1)}(\pi) R_{y}^{(2)}\left(-\frac{\pi}{2}\right)\left(M_{x}^{(1)}\right)^{-1}$ using Euler's theorem and we find $A_{\text {alt2 }}^{\mathrm{st} \rightarrow \text { Bell }}=R_{y}^{(1)}\left(-\frac{\pi}{2}\right) R_{y}^{(2)}\left(-\frac{\pi}{2}\right) M_{x}^{(1)} R_{z}^{(1)}(\pi) R_{y}^{(1)}\left(-\frac{\pi}{2}\right) R_{y}^{(2)}\left(\frac{\pi}{2}\right)$.

\subsection{The CNOT-gate}

The CNOT-gate is given by

$$
\mathrm{CNOT}^{(1,2)}=\left(\begin{array}{llll}
1 & 0 & 0 & 0 \\
0 & 1 & 0 & 0 \\
0 & 0 & 0 & 1 \\
0 & 0 & 1 & 0
\end{array}\right) .
$$

In order to find the optimal (shortest-sequence) decomposition of the CNOT-gate in terms of the (SWAP) ${ }^{\alpha}$ and B-gate, we again use the protocol from section 2 and proceed in the same way as for $U^{\text {st } \rightarrow \text { Bell }}$ in the previous subsection. We first calculate $K_{1}, A$ and $K_{2}$, then decompose each of these matrices into (SWAP) ${ }^{\alpha}$ or B-operations plus single-qubit rotations, and subsequently use permutation relations to optimize the number of rotations.

Starting with step 1 in section 2.1, we find for the representation of $\mathrm{CNOT}^{(1,2)}$ in the magic basis,

$$
\mathrm{CNOT}_{M B}^{(1,2)}=\frac{1}{2}\left(\begin{array}{cccc}
1 & \mathrm{i} & -1 & \mathrm{i} \\
-\mathrm{i} & 1 & -\mathrm{i} & -1 \\
-1 & \mathrm{i} & 1 & \mathrm{i} \\
-\mathrm{i} & -1 & -\mathrm{i} & 1
\end{array}\right)
$$

and the eigenvalues and corresponding eigenvectors of $\left(\mathrm{CNOT}_{M B}^{(1,2)^{T}} \mathrm{CNOT}_{M B}^{(1,2)}\right)$ are given by

$$
\begin{gathered}
\mu_{1}=\mu_{2}=1, \quad\left|a_{1}\right\rangle=\frac{1}{\sqrt{2}}\left(\begin{array}{c}
0 \\
-1 \\
0 \\
1
\end{array}\right), \quad\left|a_{2}\right\rangle=\frac{1}{\sqrt{2}}\left(\begin{array}{c}
-1 \\
0 \\
1 \\
0
\end{array}\right) \\
\mu_{3}=\mu_{4}=-1, \quad\left|a_{3}\right\rangle=\frac{1}{\sqrt{2}}\left(\begin{array}{l}
1 \\
0 \\
1 \\
0
\end{array}\right) \quad\left|a_{4}\right\rangle=\frac{1}{\sqrt{2}}\left(\begin{array}{l}
0 \\
1 \\
0 \\
1
\end{array}\right) .
\end{gathered}
$$

We now choose $\left|v_{1}\right\rangle \equiv\left|a_{3}\right\rangle,\left|v_{2}\right\rangle \equiv\left|a_{4}\right\rangle,\left|v_{3}\right\rangle \equiv\left|a_{2}\right\rangle$ and $\left|v_{4}\right\rangle \equiv\left|a_{1}\right\rangle$, and calculate the matrix $K_{2}$ from equation (10),

$$
K_{2}^{\mathrm{CNOT}}=\frac{1}{\sqrt{2}}\left(\begin{array}{cccc}
1 & 0 & -1 & 0 \\
0 & 1 & 0 & -1 \\
1 & 0 & 1 & 0 \\
0 & 1 & 0 & 1
\end{array}\right) .
$$


Next, we calculate the matrix $A$ from equations (11) and (1). Choosing $\lambda_{1}=\lambda_{2}=1, \lambda_{3}=$ $\lambda_{4}=\mathrm{i}$, we find the solution $\alpha=\pi / 4, \beta=\gamma=0$ and hence

$$
A^{\mathrm{CNOT}}=\frac{1}{\sqrt{2}}\left(\begin{array}{llll}
1 & 0 & 0 & \mathrm{i} \\
0 & 1 & \mathrm{i} & 0 \\
0 & \mathrm{i} & 1 & 0 \\
\mathrm{i} & 0 & 0 & 1
\end{array}\right) .
$$

Finally, we obtain $K_{1}$ from equation (12),

$$
K_{1}^{\mathrm{CNOT}}=\frac{1}{2}\left(\begin{array}{cccc}
1 & -\mathrm{i} & 1 & -\mathrm{i} \\
-\mathrm{i} & 1 & -\mathrm{i} & 1 \\
-\mathrm{i} & -1 & \mathrm{i} & 1 \\
-1 & -\mathrm{i} & 1 & \mathrm{i}
\end{array}\right) .
$$

We now decompose each of the matrices $K_{2}^{\mathrm{CNOT}}, A^{\mathrm{CNOT}}$ and $K_{1}^{\mathrm{CNOT}}$ (equations (35)-(37)) into a sequence of single-qubit rotations and (SWAP) ${ }^{\alpha}$-gates, using the procedure described in section 2.3. Starting with $K_{2}^{\mathrm{CNOT}}$ and comparing the right-hand sides of equations (35) and (13) for condition $(14 a)$, we obtain

$$
K_{2}^{\mathrm{CNOT}}=R_{y}^{(1)}\left(\frac{\pi}{2}\right) .
$$

Since $A^{\mathrm{CNOT}}=A^{\mathrm{st} \rightarrow \text { Bell }}$, the decompositions of these matrices are the same:

$$
A^{\mathrm{CNOT}}=R_{x}^{(1)}(\pi) \sqrt{\mathrm{SWAP}} R_{x}^{(1)}(\pi) \sqrt{\mathrm{SWAP}} .
$$

Finally, the decomposition of the matrix $K_{1}^{\mathrm{CNOT}} \in S U(2) \otimes S U(2)$ is obtained using equation (15), and we find,

$$
K_{1}^{\mathrm{CNOT}}=R_{y}^{(1)}\left(-\frac{\pi}{2}\right) R_{x}^{(1)}\left(\frac{\pi}{2}\right) R_{x}^{(2)}\left(\frac{\pi}{2}\right)
$$

The total decomposition of $\mathrm{CNOT}^{(1,2)}$ is then given by,

$$
\begin{aligned}
\mathrm{CNOT}_{(\mathrm{SWAP})}^{(1,2)} & =K_{1}^{\mathrm{CNOT}} A^{\mathrm{CNOT}} K_{2}^{\mathrm{CNOT}} \\
& =R_{y}^{(1)}\left(-\frac{\pi}{2}\right) R_{x}^{(2)}\left(\frac{\pi}{2}\right) R_{x}^{(1)}\left(-\frac{\pi}{2}\right) \sqrt{\operatorname{SWAP}} R_{x}^{(1)}(\pi) \sqrt{\operatorname{SWAP}} R_{y}^{(1)}\left(\frac{\pi}{2}\right) .
\end{aligned}
$$

The number of operations in equation (41) cannot be further reduced by applying commutation relations or Euler's theorem. Including possible permutations of rotation angles, we obtain the general form of equation (41), ${ }^{10}$

$\operatorname{CNOT}_{\left(\mathrm{SWAP}^{\alpha}\right.}^{(1,2)}=R_{y}^{(1)}\left(\sigma \frac{\pi}{2}\right) R_{x}^{(i)}\left(\sigma^{\prime} \frac{\pi}{2}\right) R_{x}^{(j)}\left(\sigma^{\prime \prime} \frac{\pi}{2}\right) \sqrt{\operatorname{SWAP}} R_{x}^{(i)}(\pi) \sqrt{\operatorname{SWAP}} R_{y}^{(1)}\left(-\sigma \frac{\pi}{2}\right)$

for $\left(i, j, \sigma^{\prime}, \sigma^{\prime \prime}\right) \in\{(1,2,-1,-\sigma),(2,1, \sigma, 1)\}$, with $\sigma= \pm 1$. Using the B-gate, the shortestsequence implementation of the $\mathrm{CNOT}^{(1,2)}$-gate is given by,

$\mathrm{CNOT}_{\mathrm{B}}^{(1,2)}=R_{y}^{(1)}\left(\sigma \frac{\pi}{2}\right) R_{x}^{(1)}\left(\sigma^{\prime} \frac{\pi}{2}\right) R_{x}^{(2)}\left(-\sigma^{\prime} \sigma \frac{\pi}{2}\right) B\left(\sigma^{\prime} \frac{\pi}{2}, \sigma^{\prime} \frac{\pi}{2}\right) R_{y}^{(1)}\left(-\sigma \frac{\pi}{2}\right)$,

for $\sigma, \sigma^{\prime}= \pm 1$. The sequences (30), (31), (42) and (43) for implementing $U^{\text {st } \rightarrow \text { Bell }}$ and the CNOT-gate are building blocks for the implementation of quantum operations for three or more qubits [16].

${ }^{10}$ This decomposition corresponds to the experimental setup in [24] in which only rotations in the $(x, y)$-plane are available. An alternative implementation using $z$-rotations is given in [7], see equation (8). 


\subsection{The quantum Fourier transform}

The quantum Fourier transform (QFT) lies at the heart of Shor's factoring algorithm [17] and is given by (for two qubits) [18],

$$
\mathcal{F}=\frac{1}{2}\left(\begin{array}{cccc}
1 & 1 & 1 & 1 \\
1 & \mathrm{i} & -1 & -\mathrm{i} \\
1 & -1 & 1 & -1 \\
1 & -\mathrm{i} & -1 & \mathrm{i}
\end{array}\right) .
$$

So far, the QFT has been implemented in an NMR system [19], using ion qubits [20] and using phononic qubits [21], but not yet with solid-state qubits. $\mathcal{F}^{2}=\mathrm{CNOT}^{(2,1)}$ is a maximally entangling gate, but $\mathcal{F}$ itself is not ${ }^{11}$. In order to find the shortest sequence of operations required to implement $\mathcal{F}$, we thus cannot use our optimization protocol for gates with maximally entangling capacity (as defined and described in section 2.2), but need to use the method for general gates $U \in S U$ (4) outlined in section 2.1. To this end, we use the decomposition of $\mathcal{F}$ into the controlled phase gate $C P$ plus two Hadamard gates proposed by Coppersmith et al [22],

$$
\mathcal{F}=\operatorname{SWAP} \cdot H^{(2)} \cdot C P \cdot H^{(1)}
$$

with

$$
\begin{aligned}
H^{(2)} & =\frac{1}{\sqrt{2}}\left(\begin{array}{cccc}
1 & 1 & 0 & 0 \\
1 & -1 & 0 & 0 \\
0 & 0 & 1 & 1 \\
0 & 0 & 1 & -1
\end{array}\right) \\
C P & =\left(\begin{array}{llll}
1 & 0 & 0 & 0 \\
0 & 1 & 0 & 0 \\
0 & 0 & 1 & 0 \\
0 & 0 & 0 & \mathrm{i}
\end{array}\right) \\
H^{(1)} & =\frac{1}{\sqrt{2}}\left(\begin{array}{cccc}
1 & 0 & 1 & 0 \\
0 & 1 & 0 & 1 \\
1 & 0 & -1 & 0 \\
0 & 1 & 0 & -1
\end{array}\right) .
\end{aligned}
$$

The Hadamard gates $H^{(1)}$ and $H^{(2)}$ can be decomposed into single-qubit rotations using equation (15) and we find

$$
\begin{aligned}
H^{(i)} & =R_{x}^{(i)}(\pi) R_{y}^{(i)}\left(\frac{\pi}{2}\right) \\
& =R_{y}^{(i)}\left(-\frac{\pi}{2}\right) R_{x}^{(i)}(\pi), \quad i=1,2 .
\end{aligned}
$$

We now proceed to calculate the decomposition $K_{1} A K_{2}$ of $C P$ and to translate each of these matrices into single-qubit rotations and two-qubit interactions according to the protocol given

${ }^{11}$ The maximum concurrence obtained when applying $\mathcal{F}$ to a separable quantum state $\in S U(2) \otimes S U(2)$ is $1 / 2$. 
in section 2. Starting with $C P_{M B}$, the representation of $C P$ in the magic basis, we obtain from equations $(46 b),(8)$ and (2),

$$
C P_{M B}=\frac{1}{2}\left(\begin{array}{cccc}
1+\mathrm{i} & 0 & 0 & 1+\mathrm{i} \\
0 & 2 & 0 & 0 \\
0 & 0 & 2 & 0 \\
-1-\mathrm{i} & 0 & 0 & 1+\mathrm{i}
\end{array}\right)
$$

The eigenvalues and eigenvectors of $C P_{M B}^{T} C P_{M B}$ are given by

$$
\begin{aligned}
& \mu_{1}=\mu_{2}=\mathrm{i}, \quad\left|a_{1}\right\rangle=\left(\begin{array}{l}
1 \\
0 \\
0 \\
0
\end{array}\right), \quad\left|a_{2}\right\rangle=\left(\begin{array}{l}
0 \\
0 \\
0 \\
1
\end{array}\right) \\
& \mu_{3}=\mu_{4}=1, \quad\left|a_{3}\right\rangle=\left(\begin{array}{l}
0 \\
1 \\
0 \\
0
\end{array}\right) \quad\left|a_{4}\right\rangle=\left(\begin{array}{l}
0 \\
0 \\
1 \\
0
\end{array}\right) \text {. }
\end{aligned}
$$

By choosing $\left|v_{i}\right\rangle \equiv\left|a_{i}\right\rangle$ for $i=1, \ldots 4$ and using equation (10), we obtain the matrix $K_{2}^{C P}$ :

$$
\begin{aligned}
K_{2}^{C P} & =\frac{1}{2}\left(\begin{array}{cccc}
1 & \mathrm{i} & -\mathrm{i} & 1 \\
1 & -\mathrm{i} & -\mathrm{i} & -1 \\
1 & \mathrm{i} & \mathrm{i} & -1 \\
1 & -\mathrm{i} & \mathrm{i} & 1
\end{array}\right) \\
& =R_{y}^{(1)}\left(\frac{\pi}{2}\right) R_{y}^{(2)}\left(\frac{\pi}{2}\right) R_{z}^{(1)}\left(\frac{\pi}{2}\right) R_{z}^{(2)}\left(-\frac{\pi}{2}\right) \\
& =R_{x}^{(1)}\left(\frac{\pi}{2}\right) R_{y}^{(1)}\left(\frac{\pi}{2}\right) R_{x}^{(2)}\left(-\frac{\pi}{2}\right) R_{y}^{(2)}\left(\frac{\pi}{2}\right),
\end{aligned}
$$

where we have used equation (15) to obtain the decomposition of $K_{2}^{C P}$. Assigning $\lambda_{1}=$ $\lambda_{2}=\sqrt{i}$ and $\lambda_{3}=\lambda_{4}=1$, we obtain from equation (11) the solution $\alpha=\pi / 8$ and $\beta=\gamma=0$, and thus from equation (1),

$$
\begin{aligned}
A^{C P} & =\left(\begin{array}{cccc}
\cos \left(\frac{\pi}{8}\right) & 0 & 0 & \mathrm{i} \sin \left(\frac{\pi}{8}\right) \\
0 & \cos \left(\frac{\pi}{8}\right) & \mathrm{i} \sin \left(\frac{\pi}{8}\right) & 0 \\
0 & \mathrm{i} \sin \left(\frac{\pi}{8}\right) & \cos \left(\frac{\pi}{8}\right) & 0 \\
i \sin \left(\frac{\pi}{8}\right) & 0 & 0 & \cos \left(\frac{\pi}{8}\right)
\end{array}\right) \\
& =R_{x}^{(1)}(\pi)(\mathrm{SWAP})^{\frac{1}{4}} \mathbf{R}_{\mathrm{x}}^{(1)}(\pi)(\mathrm{SWAP})^{\frac{1}{4}} .
\end{aligned}
$$

The decomposition (51) of $A^{C P}$ is obtained by calculating the concurrence (see equation (A.5)) of the column vectors of $A^{C P}$, which yields $C=4 \cos ^{2}(\pi / 8) \sin ^{2}(\pi / 8)=1 / 2$. Following the method outlined in section 2.3, we then calculate the corresponding time $t^{*}$ such that $(\mathrm{SWAP})^{\alpha\left(t^{*}\right)}$ yields the same value $1 / 2$ of the concurrence (amount of entanglement) when applied to the standard basis vectors. We find that two applications of the (SWAP) $)^{\frac{1}{4}}$-interaction, each followed by a rotation of the first qubit over $\pi$ around the $x$-axis (which interchanges the first and the third row, and the second and the fourth row of the matrix to which it is applied) yield a decomposition of the matrix (50). 
Having found the matrix $A^{C P}$, the matrix $K_{1}^{C P}$ and its decomposition can be calculated from equations (12) and (15), respectively,

$$
\begin{aligned}
K_{1}^{C P} & =\frac{1}{2}\left(\begin{array}{cccc}
1 & 1 & 1 & 1 \\
-\mathrm{i}^{\frac{1}{4} \mathrm{i} \pi} & \mathrm{i}^{\frac{1}{4} \mathrm{i} \pi} & -\mathrm{ie}^{\frac{1}{4} \mathrm{i} \pi} & \mathrm{ie}^{\frac{1}{4} \mathrm{i} \pi} \\
\mathrm{ie}^{\frac{1}{4} \mathrm{i} \pi} & \mathrm{ie}^{\frac{1}{4} \mathrm{i} \pi} & -\mathrm{ie}^{\frac{1}{4} \mathrm{i} \pi} & -\mathrm{ie}^{\frac{1}{4} \mathrm{i} \pi} \\
\mathrm{i} & -\mathrm{i} & -\mathrm{i} & \mathrm{i}
\end{array}\right) \\
& =R_{z}^{(2)}\left(\frac{3 \pi}{4}\right) R_{y}^{(2)}\left(-\frac{\pi}{2}\right) R_{z}^{(1)}\left(-\frac{\pi}{4}\right) R_{y}^{(1)}\left(-\frac{\pi}{2}\right) \\
& =R_{y}^{(1)}\left(-\frac{\pi}{2}\right) R_{x}^{(1)}\left(-\frac{\pi}{4}\right) R_{y}^{(2)}\left(-\frac{\pi}{2}\right) R_{x}^{(2)}\left(\frac{3 \pi}{4}\right) .
\end{aligned}
$$

The decomposition of the quantum Fourier transform $\mathcal{F}$ is then given by

$$
\begin{aligned}
\mathcal{F}= & \operatorname{SWAP}_{x}^{(2)}\left(-\frac{\pi}{4}\right) R_{y}^{(1)}\left(-\frac{\pi}{2}\right) R_{x}^{(1)}\left(\frac{3 \pi}{4}\right)(\mathrm{SWAP})^{\frac{1}{4}} R_{x}^{(1)}(\pi) \\
& \times(\mathrm{SWAP})^{\frac{1}{4}} R_{x}^{(1)}\left(-\frac{\pi}{2}\right) R_{x}^{(2)}\left(-\frac{\pi}{2}\right) R_{y}^{(2)}\left(\frac{\pi}{2}\right) \\
= & R_{x}^{(1)}\left(-\frac{\pi}{4}\right) R_{y}^{(2)}\left(-\frac{\pi}{2}\right) R_{x}^{(2)}\left(\frac{3 \pi}{4}\right)(\mathrm{SWAP})^{\frac{5}{4}} R_{x}^{(1)}(\pi) \\
& \times(\mathrm{SWAP})^{\frac{1}{4}} R_{x}^{(1)}\left(-\frac{\pi}{2}\right) R_{x}^{(2)}\left(-\frac{\pi}{2}\right) R_{y}^{(2)}\left(\frac{\pi}{2}\right) .
\end{aligned}
$$

Using commutation relations to minimize the number of rotations in equation (53), we obtain the decomposition of $\mathcal{F}$ that contains the smallest number of (SWAP) ${ }^{\alpha}$ - and single-qubit gates,

$\mathcal{F}_{(\mathrm{SWAP})^{\alpha}}=R_{y}^{(2)}\left(-\frac{\pi}{2}\right) R_{x}^{(2)}\left(\frac{\pi}{4}\right)(\mathrm{SWAP})^{\frac{5}{4}} R_{x}^{(1)}(\pi)(\mathrm{SWAP})^{\frac{1}{4}} R_{x}^{(2)}\left(-\frac{3 \pi}{4}\right) R_{y}^{(2)}\left(\frac{\pi}{2}\right)$,

and analogously we find for the optimal decomposition of $\mathcal{F}$ using the B-gate ${ }^{12}$,

$$
\begin{aligned}
\mathcal{F}_{B}=R_{y}^{(2)}(- & \left.\frac{\pi}{2}\right) B\left(\frac{\pi}{4}, \frac{5 \pi}{4}\right) R_{x}^{(1)}\left(\frac{\pi}{2}\right) R_{x}^{(2)}\left(\frac{\pi}{2}\right) \\
& \times B\left(-\frac{\pi}{2}, \frac{\pi}{2}\right) R_{x}^{(1)}\left(\frac{3 \pi}{4}\right) R_{x}^{(2)}\left(\frac{3 \pi}{4}\right) R_{y}^{(2)}\left(\frac{\pi}{2}\right) .
\end{aligned}
$$

$\mathcal{F}_{(\mathrm{SWAP})^{\alpha}}$ and $\mathcal{F}_{B}$ also give alternative implementations of the $\mathrm{CNOT}^{(2,1)}$ gate, which consist of applying the sequences (54) or (55) twice. These, however, are longer than the sequences obtained by optimizing CNOT directly (equations (42) and (43)), since they do not implement the transformation from standard to maximally entangled basis with the least number of two-qubit operations.

\section{Application to electron spin qubits and superconducting flux qubits}

For solid-state qubits, implementation of two-qubit quantum gates in an efficient way is important because of their short coherence times. In this section we discuss the implementation of the above results for electron spin qubits and superconducting flux qubits and estimate relevant time scales. An electron spin qubit consists of a single electron confined in a quantum

\footnotetext{
12 It is interesting to note that a 'swapped QFT' can be implemented for flux qubits using three operations less than the QFT itself: $(\mathrm{SWAP} \cdot \mathcal{F})_{\text {flux }}=R_{y}^{(1)}\left(-\frac{\pi}{2}\right) R_{x}^{(1)}\left(-\frac{3 \pi}{4}\right) B\left(\frac{\pi}{4}, \frac{\pi}{4}\right) R_{x}^{(2)}\left(-\frac{3 \pi}{4}\right) R_{y}^{(2)}\left(\frac{\pi}{2}\right)$. When trying to reduce operational costs, it is thus advantageous to use SWAP $\mathcal{F}$ instead of $\mathcal{F}$ in quantum protocols for flux qubits.
} 
dot, a small island in a semiconductor structure that can be filled with electrons in a controlled way [23]. The qubit is encoded in the spin degree of freedom. Single qubit rotations can be generated by applying an electron spin resonance (ESR) pulse to the electron which is described by the evolution operator $U_{R}(t)=\exp \left[-(i / \hbar) \int_{0}^{t} \mathcal{H}_{R}(\tau) \mathrm{d} \tau\right]$, with $\mathcal{H}_{R}(t)=-\frac{1}{2} \hbar \gamma \vec{B}(t) \cdot \vec{\sigma}$. Rotations of qubits 1 and 2 are then represented as:

$$
R_{n}^{(1)}(\beta)=\mathrm{e}^{-\frac{1}{2} \mathrm{i} \beta \vec{n} \cdot \vec{\sigma} \otimes I} \quad \text { and } \quad R_{n}^{(2)}(\beta)=\mathrm{e}^{-\frac{1}{2} \mathrm{i} \beta I \otimes \vec{n} \cdot \vec{\sigma}}
$$

with $\beta(t) \equiv-\gamma \int_{0}^{t} B(\tau) \mathrm{d} \tau, \gamma$ the gyromagnetic ratio, $B(t)$ the magnetic ESR field (applied in the direction perpendicular to the Zeeman-splitting field), $\vec{n} \equiv(\sin \theta \cos \phi, \sin \theta \sin \phi, \cos \theta)$ a unit vector on the Bloch sphere $[\theta \in[0, \pi), \phi \in[0,2 \pi)], \vec{\sigma} \equiv\left(\sigma_{x}, \sigma_{y}, \sigma_{z}\right)$ and $I$ the identity matrix. Two-qubit interactions arise from the Heisenberg exchange interaction, so that the $(\mathrm{SWAP})^{\alpha}$-gate (equation (17)) is the natural two-qubit gate for electron spin qubits. Both single-spin rotations and $\sqrt{\mathrm{SWAP}}$-operations have recently been demonstrated for spin qubits [24, 25], and typical times are $t_{\text {rot }} \sim 100 \mathrm{~ns}$ (for magnetic fields of $1 \mathrm{mT}$ ) and $t_{\sqrt{\mathrm{swap}}} \sim 180 \mathrm{ps}$.

Flux qubits [26] consist of a superconducting loop interrupted by three or four Josephson junctions. The qubit basis states consist of the direction of the current that is circulating around the loop and single qubit rotations are generated by applying resonant microwave radiation. These rotations are described by the same expression (56) as for spin qubits with $\beta \rightarrow \frac{1}{\hbar} \int_{0}^{t} \Omega_{j}(\tau) \cos \phi_{j}(\tau) \mathrm{d} \tau$ for $\sigma_{x}$ (rotations around the $x$-axis) and $\beta \rightarrow-\frac{1}{\hbar} \int_{0}^{t} \Omega_{j}(\tau) \sin \phi_{j}(\tau) \mathrm{d} \tau$ for $\sigma_{y}$ (rotations around the $y$-axis), $j=1,2$. Here $\Omega_{j}(\tau)$ and $\phi_{j}(\tau)$ denote the amplitude and phase of the applied microwave signal respectively. Measured Rabi oscillations are of the order of 1-10 ns [27]. Various proposals to achieve a tunable coupling mechanism for flux qubits have recently been put forward [14, 28, 29] and/or realized [30]. In each of these the proposed two-qubit gates are equivalent to the B-gate [31]. In [14], the creation of controllable coupling between two detuned flux qubits via the quantum inductance of a third flux qubit is suggested, as described by the Hamiltonian (18). Predicted $\mathrm{B}$-gate operation times for this system range from $\sim 10 \mathrm{~ns}$ [14] down to $\sim 2 \mathrm{~ns}[28]$.

In practice, manipulating qubits introduces decoherence. The single-qubit decoherence time $T_{2}$ has not been measured yet for electron spins, but recent experiments show that singlespin Rabi oscillations remain visible for up to $1 \mu \mathrm{s}$ (where each oscillation takes $\sim 100 \mathrm{~ns}$ ) [24] and ensemble decoherence times $T_{2}^{*}>1 \mu \mathrm{s}$ [25], so that $T_{2}$ is expected to be $\geqslant 1 \mu \mathrm{s}$. For flux qubits, measurements of $T_{2}$ range from 15 ns [27] to a few $\mu$ s [32], while a Rabi oscillation requires $\sim 5 \mathrm{~ns}$. $T_{2}$ is thus sufficiently long to observe at least a few Rabi oscillations. It is unknown, however, to what degree decoherence will affect a quantum gate operation which consists of several single qubit rotations on different qubits as well as two-qubit interactions: minimizing the total number of operations used [33] is then likely to be an essential factor for achieving high gate fidelities.

\section{Conclusion}

We have presented a systematic protocol for calculating the optimal (defined as consisting of the smallest number of single- and two-qubit operations) implementation of a desired twoqubit gate $U \in S U(4)$ in terms of a given tunable two-qubit interaction $U_{\text {int }}(t)$ and single-qubit rotations. We have illustrated the decomposition method by calculating the shortest sequence of operations required to implement the transformation from the standard basis to the Bell basis, the CNOT-gate, and the quantum Fourier transform, using either Heisenberg exchange interaction or quantum inductive coupling. The general method presented here is a useful tool to find the smallest number of operations that are needed to realize a desired two-qubit gate 
for any type of qubit, using the single- and tunable two-qubit operations that are naturally available in the qubit system.

\section{Acknowledgments}

This work has been supported by the Netherlands Organisation for Scientific Research (NWO) and by the EU's Human Potential Research Network under contract no. HPRN-CT-2002-00309 ('QUACS').

\section{Appendix}

In this appendix we calculate the concurrence of the column vectors of the matrix

$$
T \equiv A(\epsilon) R_{x}^{(1)}(\mu) R_{x}^{(2)}(v) R_{y}^{(1)}(\xi) R_{y}^{(2)}(\zeta),
$$

which is used in the proof of proposition 1 in section 2.2. By writing out equation (A.1) explicitly, we obtain

$$
\begin{aligned}
T & =\left(\begin{array}{cccc}
B_{1} & B_{2} & -\mathrm{i} B_{2}^{*} & \mathrm{i} B_{1}^{*} \\
\alpha B_{2} & \alpha B_{1} & \mathrm{i} \alpha B_{1}^{*} & -\mathrm{i} \alpha B_{2}^{*} \\
-\mathrm{i} \alpha B_{2}^{*} & \mathrm{i} \alpha B_{1}^{*} & \alpha B_{1} & \alpha B_{2} \\
\mathrm{i} B_{1}^{*} & -\mathrm{i} B_{2}^{*} & B_{2} & B_{1}
\end{array}\right)\left(\begin{array}{cccc}
C_{1} & -C_{2} & -C_{3} & C_{4} \\
C_{2} & C_{1} & -C_{4} & -C_{3} \\
C_{3} & -C_{4} & C_{1} & -C_{2} \\
C_{4} & C_{3} & C_{2} & C_{1}
\end{array}\right) \\
& =\left(\begin{array}{ccccc}
B_{1} C_{1}+B_{2} C_{2}-\mathrm{i} B_{2}^{*} C_{3}+\mathrm{i} B_{1}^{*} C_{4} & \ldots & \ldots & \ldots \\
\alpha\left(B_{2} C_{1}+B_{1} C_{2}+\mathrm{i} B_{1}^{*} C_{3}-\mathrm{i} B_{2}^{*} C_{4}\right) & \ldots & \ldots & \ldots \\
\alpha\left(-\mathrm{i} B_{2}^{*} C_{1}+\mathrm{i} B_{1}^{*} C_{2}+B_{1} C_{3}+B_{2} C_{4}\right) & \ldots & \ldots & \ldots \\
\mathrm{i} B_{1}^{*} C_{1}-\mathrm{i} B_{2}^{*} C_{2}+B_{2} C_{3}+B_{1} C_{4} & \ldots & \ldots & \ldots
\end{array}\right),
\end{aligned}
$$

with

$$
\begin{aligned}
B_{1} & \equiv \cos \frac{\mu}{2} \cos \frac{\nu}{2}-\mathrm{i} \sin \frac{\mu}{2} \sin \frac{\nu}{2} \\
B_{2} & \equiv \sin \frac{\mu}{2} \cos \frac{\nu}{2}-\mathrm{i} \cos \frac{\mu}{2} \sin \frac{\nu}{2} \\
C_{1} & \equiv \cos \frac{\xi}{2} \cos \frac{\zeta}{2} \\
C_{2} & \equiv \cos \frac{\xi}{2} \sin \frac{\zeta}{2} \\
C_{3} & \equiv \sin \frac{\xi}{2} \cos \frac{\zeta}{2} \\
C_{4} & \equiv \sin \frac{\xi}{2} \sin \frac{\zeta}{2} \\
\alpha & \equiv \exp \left(-\mathrm{i} \frac{\pi}{2} \epsilon\right) .
\end{aligned}
$$

The concurrence $C$ of each of the column vectors $\left|w_{i}\right\rangle$ of $T$ is found by calculating the square roots $\lambda_{1} \geqslant \lambda_{2} \geqslant \lambda_{3} \geqslant \lambda_{4}$ of the eigenvalues of the matrix

$$
M \equiv \rho_{i}\left(\sigma_{y} \otimes \sigma_{y}\right) \rho_{i}^{*}\left(\sigma_{y} \otimes \sigma_{y}\right),
$$

with $\rho_{i} \equiv\left|w_{i}\right\rangle\left\langle w_{i}\right|, i=1, \ldots, 4$, and substituting

$$
C \equiv \max \left\{\lambda_{1}-\lambda_{2}-\lambda_{3}-\lambda_{4}, 0\right\} .
$$


The conditions on $\epsilon, \mu, v, \xi$ and $\zeta$ in equation (14) are then obtained by evaluating $C=1$. The resulting expressions are in general lengthy and therefore not given here. As an example, consider the special case $\xi=\zeta=0$. In this case, $T$ (equation (A.1)) reduces to

$$
T_{\xi=\zeta=0}=\left(\begin{array}{cccc}
B_{1} & B_{2} & -\mathrm{i} B_{2}^{*} & \mathrm{i} B_{1}^{*} \\
\alpha B_{2} & \alpha B_{1} & \mathrm{i} \alpha B_{1}^{*} & -\mathrm{i} \alpha B_{2}^{*} \\
-\mathrm{i} \alpha B_{2}^{*} & \mathrm{i} \alpha B_{1}^{*} & \alpha B_{1} & \alpha B_{2} \\
\mathrm{i} B_{1}^{*} & -\mathrm{i} B_{2}^{*} & B_{2} & B_{1}
\end{array}\right) .
$$

We calculate the concurrence of the last column vector $\left|w_{4}\right\rangle$ of equation (A.6) (the same result is obtained for the other column vectors). Let

$$
\rho=\left|w_{4}\right\rangle\left\langle w_{4}\right|=\frac{1}{2}\left(\begin{array}{cccc}
\left|B_{1}\right|^{2} & -\alpha^{*} B_{1}^{*} B_{2} & \mathrm{i} \alpha^{*} B_{1}^{*} B_{2}^{*} & \mathrm{i}\left(B_{1}^{*}\right)^{2} \\
-\alpha B_{1} B_{2}^{*} & \left|B_{2}\right|^{2} & -\mathrm{i}\left(B_{2}^{*}\right)^{2} & -\mathrm{i} \alpha B_{1}^{*} B_{2}^{*} \\
-\mathrm{i} \alpha B_{1} B_{2} & \mathrm{i} B_{2}^{2} & \left|B_{2}\right|^{2} & \alpha B_{1}^{*} B_{2} \\
-i B_{1}^{2} & \mathrm{i} \alpha^{*} B_{1} B_{2} & \alpha^{*} B_{1} B_{2}^{*} & \left|B_{1}\right|^{2}
\end{array}\right) .
$$

Then $M$ (equation (A.4)) is given by

$$
\frac{1}{2}\left(\begin{array}{cccc}
\left|B_{1}\right|^{2} P & -B_{1}^{*} B_{2} Q & \mathrm{i} B_{1}^{*} B_{2}^{*} Q & \mathrm{i}\left(B_{1}^{*}\right)^{2} P \\
-B_{1} B_{2}^{*} Q & \left|B_{2}\right|^{2} R & -\mathrm{i}\left(B_{2}^{*}\right)^{2} R & -\mathrm{i} B_{1}^{*} B_{2}^{*} Q \\
-\mathrm{i} B_{1} B_{2} Q & \mathrm{i} B_{2}^{2} R & \left|B_{2}\right|^{2} R & B_{1}^{*} B_{2} Q \\
-\mathrm{i} B_{1}^{2} P & \mathrm{i} B_{1} B_{2} Q & B_{1} B_{2}^{*} Q & \left|B_{1}\right|^{2} P
\end{array}\right),
$$

with

$$
\begin{aligned}
& P \equiv\left|B_{1}\right|^{2}+\left(\alpha^{*}\right)^{2}\left|B_{2}\right|^{2} \\
& Q \equiv \alpha\left|B_{1}\right|^{2}+\alpha^{*}\left|B_{2}\right|^{2} \\
& R \equiv \alpha^{2}\left|B_{1}\right|^{2}+\left|B_{2}\right|^{2} .
\end{aligned}
$$

The eigenvalues of equation (A.8) are the solutions of the equation

$\lambda^{3}\left(\lambda-2\left(\left|B_{1}\right|^{2} P+\left|B_{2}\right|^{2} R\right)\right)=0$

$$
\Leftrightarrow \quad \lambda=0 \text { or } \lambda=\left|B_{1}\right|^{4}+2 \cos (\epsilon \pi)\left|B_{1}\right|^{2}\left|B_{2}\right|^{2}+\left|B_{2}\right|^{4}>0
$$

so that

$$
C=\sqrt{\left|B_{1}\right|^{4}+2 \cos (\epsilon \pi)\left|B_{1}\right|^{2}\left|B_{2}\right|^{2}+\left|B_{2}\right|^{4}} .
$$

Finally,

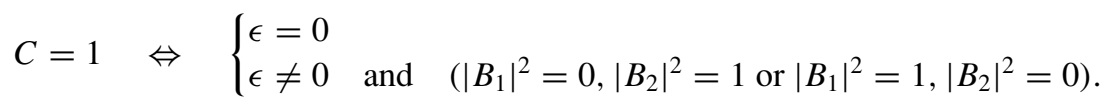

The latter solution corresponds to $\mu, \nu \in\{0, \pi, 2 \pi, 3 \pi, \ldots\}$. Equation (A.11) corresponds to the solution given in equation (14).

\section{References}

[1] Vidal G and Dawson C M 2004 Phys. Rev. A 69010301

Shende V V, Bullock S S and Markov I L 2004 Phys. Rev. A 70012310

Vatan F and Williams C 2004 Phys. Rev. A 69032315

Zhang J, Vala J, Sastry S and Whaley K B 2004 Phys. Rev. A 69042309

[2] Fan H, Roychowdhury V and Szkopek T 2005 Phys. Rev. A 72052323

[3] Zhang J et al 2004 Phys. Rev. Lett. 93020502

[4] Kraus B and Cirac J I 2001 Phys. Rev A 63062309

[5] Khaneja N and Glaser S J 2001 Chem. Phys. 26711

[6] Vidal G, Hammerer K and Cirac J I 2002 Phys. Rev. Lett. 88237902 
[7] Burkard G et al 1999 Phys. Rev. B 6011404

[8] This follows from group theory, see e.g. Rossmann W 2002 Lie Groups-An Introduction Through Linear Groups (New York: Oxford University Press) (It can also be proven by writing out $\mathrm{O}_{2}$ for a general matrix $\left.K_{2} \in S U(2) \otimes S U(2)\right)$

[9] Rezakhani A T 2004 Phys. Rev. A 70052313 (see also [3])

[10] Wootters W K 1998 Phys. Rev. Lett. 802245

[11] Barenco A et al 1995 Phys. Rev. A 523457

[12] See Zhang J, Vala J, Sastry S and Whaley K B 2003 Phys. Rev. Lett. 91027903 See e.g. Bremner M J et al 2002 Phys. Rev. Lett. 89247902

[13] de Visser R L and Blaauboer M 2006 Phys. Rev. Lett. 96246801

[14] See e.g. Niskanen A O, Nakamura Y and Tsai J-S 2006 Phys. Rev. B 73094506 (and references therein)

[15] Makhlin Y 2002 Quantum Inform. Process. 1243

[16] Bodoky F and Blaauboer M 2007 Phys. Rev. A 76052309

[17] Shor P W 1997 SIAM J. Comput. 261484

[18] Nielsen M and Chuang I 2000 Quantum Computation and Quantum Information (Cambridge: Cambridge University Press) chapter 5

[19] Vandersypen L M K et al 2000 Phys. Rev. Lett. 855452

Weinstein Y S et al 2001 Phys. Rev. Lett. 861889

See also the discussion on efficient implementation in Dorai K and Suter D 2005 Int. J. Quantum Inf. $3413-24$

[20] Chiaverini J et al 2005 Science 308997

[21] Lu C Y et al 2007 Phys. Rev. Lett. 99250504 (arXiv:quant-ph/07051684)

[22] Coppersmith D 1994 IBM Research Report No. RC19642 See also Weinstein Y S et al 2001 Phys. Rev. Lett. 861889

[23] For a recent review on few-electron quantum dots as spin qubits, see Elzerman J M et al 2005 Lect. Notes Phys. 66725

[24] Koppens F H L et al 2006 Nature 442766

[25] Petta J R et al 2005 Science 3092180

[26] Wendin G and Shumeiko V S 2005 arXiv:cond-mat/0508729 Makhlin Y, Schön G and Shnirman A 2001 Rev. Mod. Phys. 73357

[27] Chiorescu I et al 2003 Science 2991869

[28] Grajcar M et al 2006 Phys. Rev. B 74172505

[29] Plourde B L T et al 2004 Phys. Rev. B 70 140501(R)

[30] Hime T et al 2006 Science 3141427

[31] After completing this work, another experimental realization of a tunable flux qubit coupling has been reported by Plantenberg J et al 2007 Nature 447836

[32] Bertet P et al 2005 Phys. Rev. Lett. 95257002

[33] A proposal for implementing two-qubit gates in a single step by carrying out one-qubit and two-qubit operations simultaneously has been presented by Grigorenko I A and Khveshchenko D V 2005 Phys. Rev. Lett. 95110501 